\title{
Surgical Management of Drug-Induced Gingival Overgrowth (DIGO) Using A Periodontal Resective Flap Procedure: A Case Report
}

\author{
E Gamble, D Chatzopoulou and DG Gillam* \\ Queen Mary University London, London
}

Submission: October 10, 2018; Published: November 15, 2018

*Corresponding author: Gillam DG,Oral BioEngineering, Institute of Dentistry, Barts and the London School of Medicine and Dentistry, Queen Mary University London, London

\section{Abstract}

This case report describes the surgical management of a patient with Drug-induced Gingival Overgrowth (DIG0) as a result of a cyclosporin induced gingival hyperplasia. The report also highlights the importance of an extensive oral hygiene regime following the surgical procedure as well as the patient's ability to maintain an optimal plaque control.

Keywords: Surgical technique; resective flap procedure; Drug induced gingival overgrowth (DIGO); Case report; Patient compliance

\section{Introduction}

Gingival hyperplasia or Drug-induced Gingival Overgrowth (DIGO) may be caused by a variety of conditions or prescribed medications and/or therapies, for example, immuno-suppressive therapy (cyclosporin), anticonvulsants (phenytoin) and calcium channel blockers (nifedipine, amlodipine or diltiazem). According to Ellis [1] the prevalence of Gingival Overgrowth or DIGO is approximately $20 \%$ to $83 \%$ of the patients taking the medication. Of the medication prescribed $50 \%$ of DIGO is attributed to phenytoin, $30 \%$ to cyclosporin, and $10 \%$ to $20 \%$ to calcium channel blockers. Nifedipine appears to be the more prominent calcium channel blocker causing DIGO [2]. Hyperplasia of this nature is usually the result of a combination of the above-mentioned medications and a local irritant such as plaque biofilms. Plaque may be associated with dental calculus or a poorly adapted margin of a dental restoration, which may allow biofilm accumulation and maturation and therefore may impede its disruption and removal. If left untreated, this oral condition can affect the alignment of the teeth and increase the risk of developing gum disease. Gingival hyperplasia can be resolved by improving oral hygiene habits. In more severe cases, surgical treatment is necessary. It has been proposed that medication affects the normal inflammatory response with a resultant increase in gingival fibroblast proliferation and collagen production [3].

\section{Aim}

The aim of this case report was to describe a surgical technique used for the excision of a cyclosporin induced gingival hyperplasia (Figures 1 \& 2) in the anterior mandibular region (lower jaw) in order to establish a favourable anatomy for a good standard of oral hygiene by the patient. Furthermore to report on any associated changes in the periodontal parameters of probing depth and bleeding on probing following excision, adaptation of the gingival tissues and a subsequent extensive oral hygiene regime.

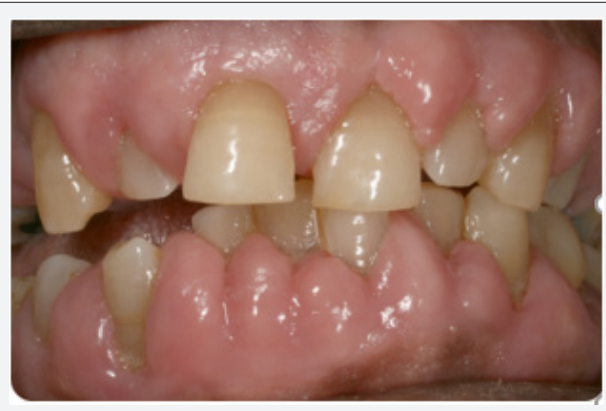

Figure 1: Pre-operative anterior view.

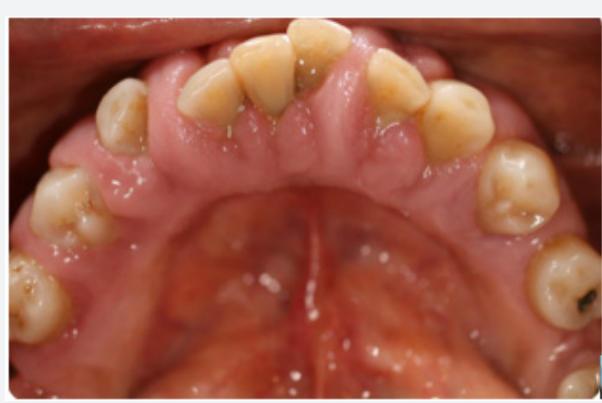

Figure 2: Pre-operative occlusal view. 


\section{Advances in Dentistry \& Oral Health}

\section{Case Report}

\section{Description}

An 81-year-old, non-smoking male with a drug induced gingival hyperplasia, presented to the postgraduate periodontal clinic. After the completion of one course of non-surgical periodontal treatment, a test site of gingival tissue was removed surgically. Local anaesthesia was obtained, and the apical extension of the gingival pockets were identified using a probe \& external bleeding points were achieved (Figure 3). A horizontal incision, joining the bleeding points, was made and all coronal tissue was removed (Figure 4). The gingival thickness of the site was assessed, and further periodontal tissue was removed via an internal bevel incision to allow for a closer adaptation of the flap to the teeth. Two full thickness mucoperiosteal flaps were raised buccally and lingually. The teeth surfaces were meticulously cleaned \& interrupted sutures (VICRYL[Polyglactin 910] 5.0) secured the remaining tissue (Figures $5 \&$ ). Further oral hygiene instructions were subsequently given to the patient.
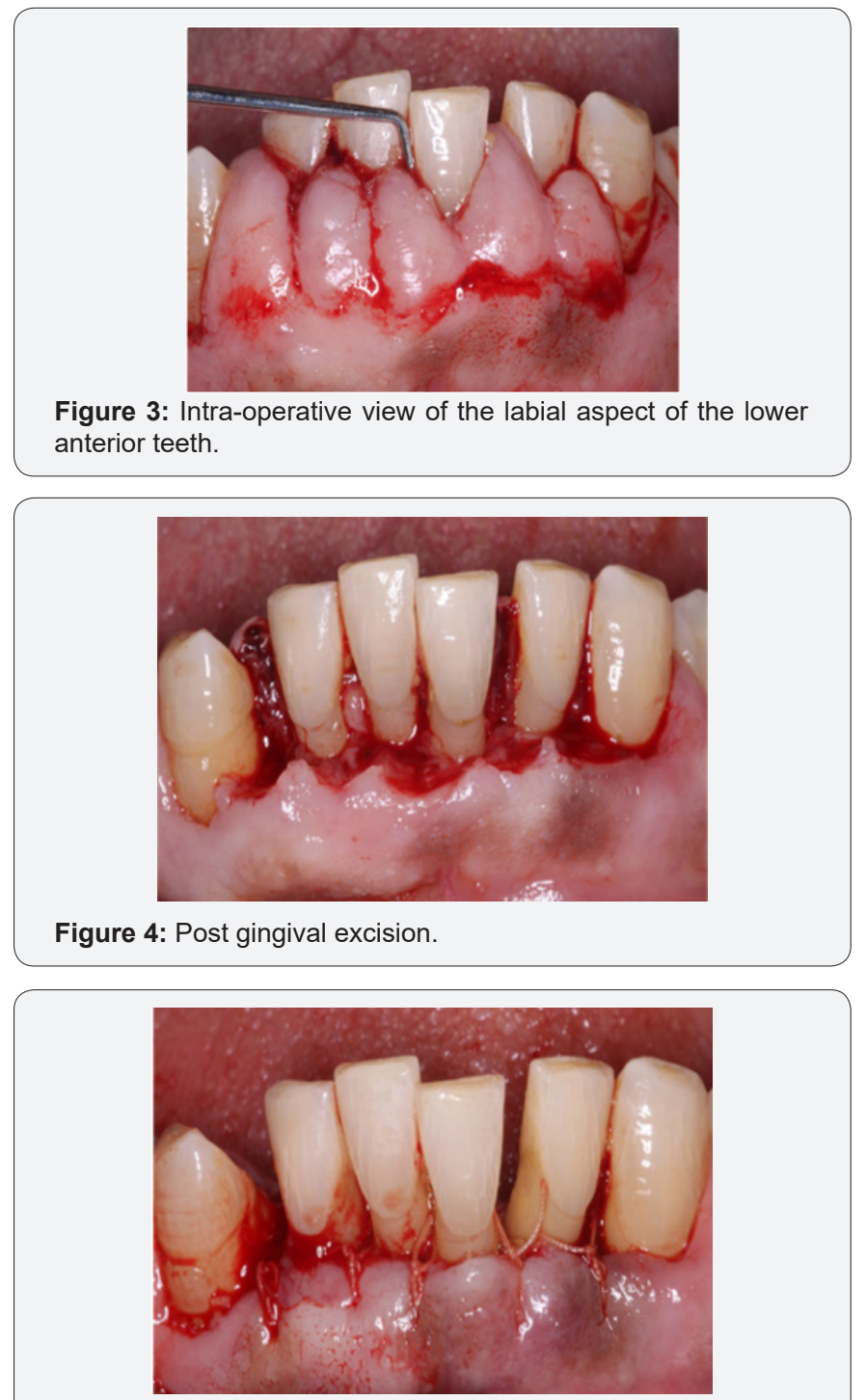

Figure 5: Immediate post-operative labial view.

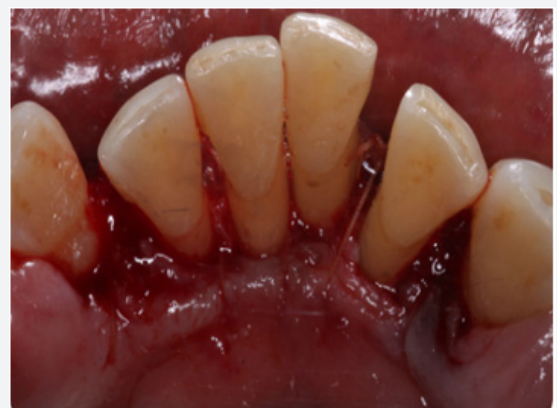

Figure 6: Immediate post-operative lingual view.

\section{Post-operative view at 1 month}

Following the surgical excision of the hyperplastic tissue and non-surgical debridement of the lower incisors it was noted that there was an overall improvement in the gingival condition with reduced probing depths and less bleeding on probing (Figures 7-10). The importance of maintaining meticulous care of the oral tissues was reinforced at this visit given that the patient was rinsing twice daily $(10 \mathrm{~mL})$ with a chlorhexidine mouthwash (Corsodyl, GlaxoSmithKline Consumer Healthcare, UK) together with a $1 \%$ chlorhexidine gel twice daily (Corsodyl, GlaxoSmithKline Consumer Healthcare, UK).

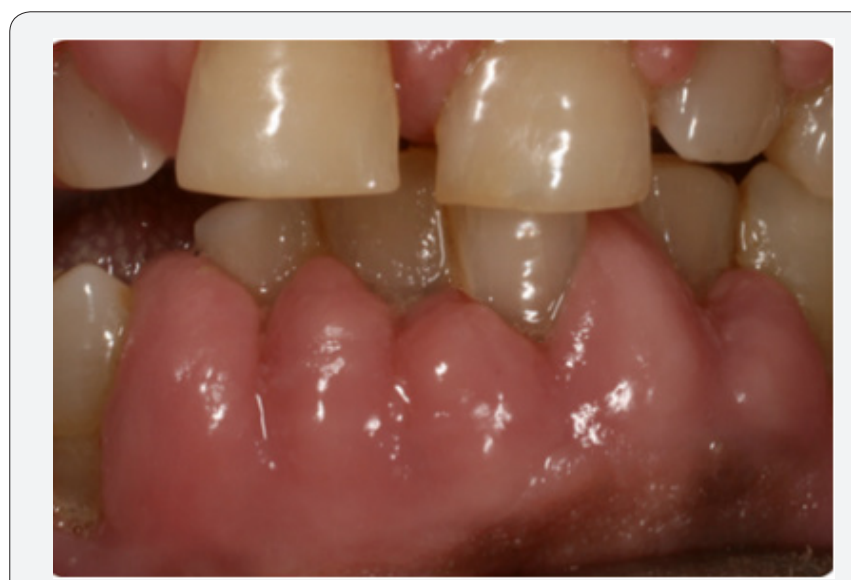

Figure 7: Pre-operative labial view.

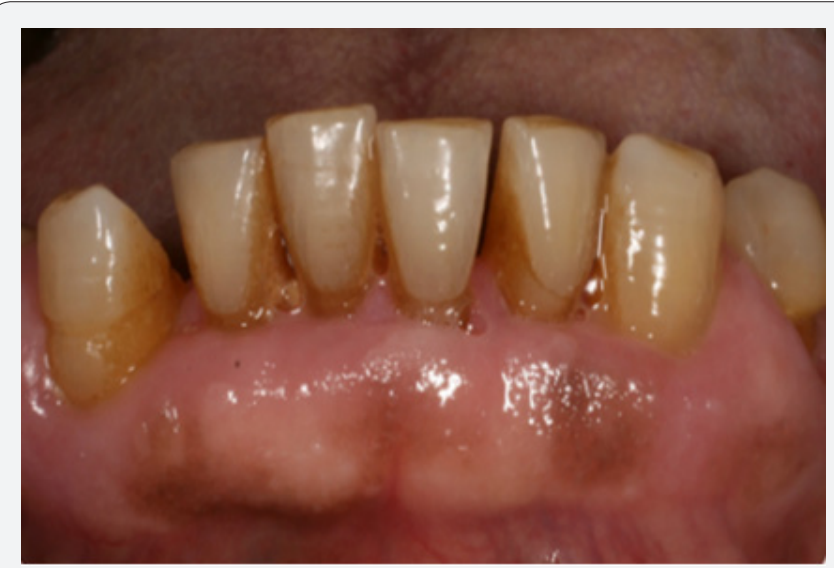

Figure 8: Post-operative labial view. 


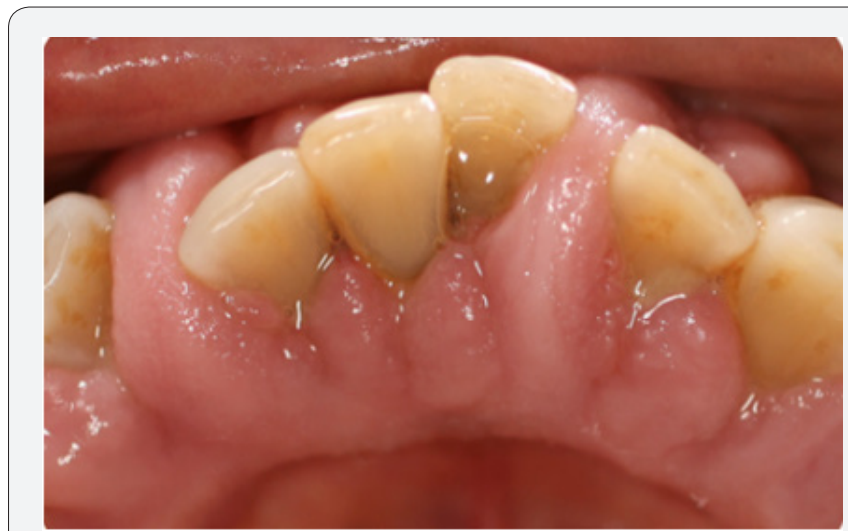

Figure 9: Pre-operative lingual view.

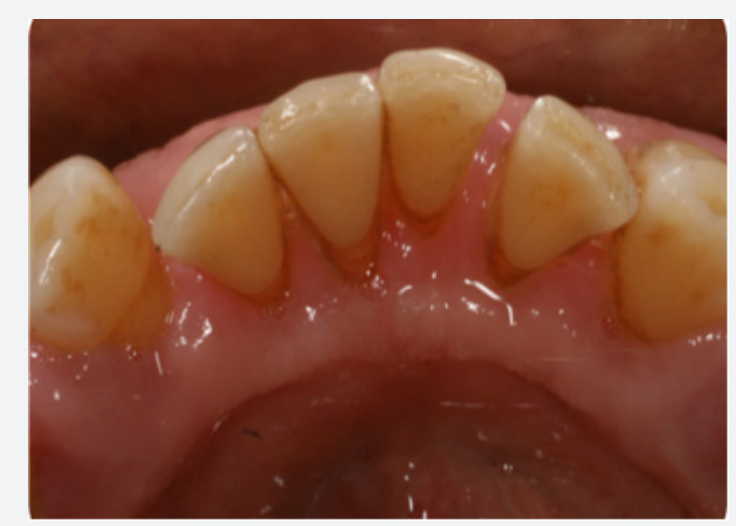

Figure 10: Post-operative lingual view.

\section{Conclusion}

In conclusion, the maintenance of the patient's postsurgical gingival health \& the resultant aesthetic appearance were related to

a. the surgical technique used and

b. the patient's compliance in maintaining optimum plaque control.

The maintenance of an optimal level of oral hygiene is therefore essential in the preservation of the gingival health following the surgical excision of the hyperplastic tissue.

\section{References}

1. Ellis JS, Seymour RA, Steele JG, Robertson P, Butler TJ, et al. (1999) Prevalence of gingival overgrowth induced by calcium channel blockers: a community-based study. J Periodontol 70(1): 63-67.

2. Froum S (2017) Top causes of gingival enlargement and treatment options. Perio-Implant Advisory.

3. Bharti V, Bansal C (2013) Drug-induced gingival overgrowth: The nemesis of gingiva unravelled. J Indian Soc Periodontol 17(2): 182-187.

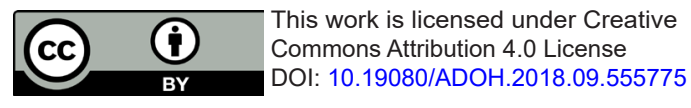

